patients with Duchenne muscular dystrophy, though $\mathrm{Dr}$ Mukoyama (Nagoya) described some changes of the myelin sheaths in the spinal roots of such patients. Bradley's studies repeating the transplantation experiments originally taken to support the neural theory gave reason to suspect that the contraction of the host muscle might be recorded from the transplanted regenerate. $\mathrm{Dr}$ Neerunjun (Royal Postgraduate Medical School) however reported studies which supported the neural theory. Dr A. C. Petenson (University of British Columbia) described chimera experiments indicating that a skeletal muscle fibre might be morphologically normal though it had $96 \%$ dystrophic nuclei. From these experiments he concluded that the cause of murine muscular dystrophy lay outside the skeletal muscle. In discussion, the question was raised whether one or two per cent of normal nuclei might provide sufficient of a putative deficient substance to cure the disease. Dr M. Rathbone (McMaster University) showed that transplantation of foetal spinal cord from dystrophic chicks could produce biochemical enzyme changes indicative of dystrophy in otherwise normal chicks.

A session was devoted to papers bearing on the vascular theory of muscular dystrophy. Neither Drs O. B. Paulson and F. Jerusalem (Mayo Clinic, Rochester) nor Dr M. D. O'Brien (University of Newcastle upon Tyne) found decreases in muscle blood flow or capillary abnormalities in Duchenne muscular dystrophy. Professor K. Kunze commented that denervated human skeletal muscle shows similar or even lower $P_{\mathrm{O}_{2}}$ values when locally measured and compared with those in Duchenne and other muscular dystrophies. No defence of the vascular theory came in discussion from $\mathrm{Dr}$ W. King Engel (National Institutes of Health, Bethesda), though he restated his theory in a later review session dealing with aetiological theories.

In sessions on the clinical management of human muscular dystrophy, Dr I. M. Siegel (University of Chicago) reviewed the results of his enthusiastic and effective treatment of muscular dystrophy with surgery and surgical appliances. Dr R. H. T. Edwards (Royal Postgraduate Medical School) described a simple dynamometer for use in clinical studies of patients with muscle weakness. Dr J. B. Peter (University of California, Los Angeles) described a controlled trial of long-term high dose corticosteroid therapy in Duchenne muscular dystrophy. Initial results indicated a fall in the serum enzyme activities, but no centain clinical improvement to date.

Other sessions dealt with normal muscle, with various forms of myopathy, malignant hyperpyrexia, and myositis, as well as the various animal models of muscular dystrophy. Biochemical studies of membrane abnormalities in myotonic conditions proved stimulating. Drs A. D. Roses and S. H. Appel (Duke University) reported various membrane abnormalities, including that of membrane protein phosphorylation in dystrophia myotonica. $\mathrm{Dr} \mathrm{R}$. M. Andiman (University of California, Los Angeles) found an increase of desmosterol in the membranes of animals rendered myotonic by various drugs, but no increase in human myotonic membranes.

Peripheral nerve studies in health and disease were fully considered in the congress. A session and a workshop were devoted to axoplasmic flow. Dr
S. Ochs (University of Indianapolis) reviewed the evidence for fast and slow rates of axoplasmic flow and $\mathrm{Dr}$ G. W. Gross (Munich) described his experiments on the garfish olfactory nerve, which led him to propound a theoretical model of the many features making up axoplasmic flow. Dr B. G. Livett (Monash University) (for Professor L. Austin) described experiments showing marked abnormalities of axoplasmic flow of proteins and lipids in the nerves of dystrophic mice. In a fruitful workshop on motor unit function, Professor E. Henneman (Harvard) described evidence that motor units have a regular and repeatable order of activation. This gave rise to considerable discussion, not all speakers being convinced by the evidence.

\title{
Mad March hares
}

from our Animal Ecology Correspondent

THE springtime behaviour of hares, normally peaceful and retiring creatures, is proverbial. During February, March and April they gang together, becoming bellicose, vocal and extrovert. The 'madness' lasts only a few weeks, beginning at the start of the breeding season but ending long before the breeding season is finished.

In a thorough study on the breeding biology of the European hare, G. A. Lincoln has brought to light some intriguing questions (J. Zool., Lond., 174, 1; 1974). The period of sexual quiescence in does lasts from September to January, and in bucks viable sperm occurs in the epidiymides from mid-December to early September. The number of sperms present reaches a high level from February to June with the maximum testosterone content in April. Pregnancies were recorded every month from January to August with the maximum number of females pregnant and the highest mean number of foetuses per pregnancy occurning in April, May and June.

Lincoln found spermatozoa in cervical smears taken from does during November and December, well before the first ovulation of the season. (In this mammal ovulation is initiated by coitus: Stieve, Zool. Anz., 148, 101; 1952.) The hormonal complexion of the hare does not allow ovulation to occur until early January, so matings earlier than this fail to result in pregnancy. Perhaps such matings have a social function providing necessary courtship and mating experience for young animals.

'March madness' is thought by
Lincoln to be a rut having the function of stimulating the resumption of breeding activity among the does. Wynne-Edwards, however, believes it to be a form of self advertisement (Animal Dispersion in Relation to Social Behaviour, Oliver and Boyd, Edinburgh, 1962). The complex chasing and boxing of the 'madness' is a social means of establishing a breeding hierarchy amongst the males, possibly identifying socially inexperienced individuals from the rest. He argues that this must happen right at the start of the breeding season so that the year's "quota" of reproduction can be assessed. Both interpretations can be correct and verification must await further study. Certainly the behaviour has little immediate physiological effect as only $16 \%$ of March madness conceptions result in hares the following spring compared with between $39 \%$ and $45 \%$ from later conceptions.

The suggestion that a mechanism exists whereby young hares gain sexual experience before actually breeding has a parallel in at least one other species of mammal. Young male baboons mate with females at the very start of their oestrus cycles when the females' receptivity and chance of pregnancy is low. Towards the end of their cycles, females mate with older and more experienced males when their receptivity is high. Most pregnancies result from these late matings (Washburn and DeVore, Scient. Am,. June 1961). Perhaps even hares have a society with complex rules of conduct which offers to instruct young initiates in its mysteries. 